\title{
Robust Surface Registration Using Optimal Mass Transport and Teichmüller Mapping
}

\begin{abstract}
Surface registration plays a significant role in computer vision and engineering fields. One of the most challenging problems in surface registration, however, is to obtain a unique bijective registration for surfaces with large deformations and landmarks constraints. In this work, a novel surface registration framework is proposed to tackle this problem using optimal mass transport mapping (OMTMap) and Teichmüller mapping (T-Map). All metric surfaces with the disk topology are mapped to the planar disk using OMT-Map, which avoids huge area distortion, thus rendering our method more robust. A landmarkconstrained T-Map is then computed between two planar disks such that the maximal conformality distortion is minimized while the landmarks are matched. Compared with existing surface registration methods, our method is more advantageous in enforcing the robustness by avoiding large area distortion, and producing diffeomorphisms with all landmarks matched consistently. Numerical experiments on various surfaces demonstrate the efficiency, robustness of our method.
\end{abstract}

Keywords: Surface registration, large deformations, landmark constraints, optimal mass transport, Teichmüller mapping

\section{Introduction}

The past decade has witnessed a remarkable growth of interest and research efforts in 3D surface registration, ranging from computer vision field [1-3] to medical imaging field $[4,5]$. Given two metric surfaces $\left(S_{1}, g_{1}\right)$ and $\left(S_{2}, g_{2}\right)$ with Riemannian metrics, the surface registration problem is to find an optimal oneto-one correspondence mapping $f: S_{1} \rightarrow S_{2}$ between the surfaces.

Landmark-free surface registration has been studied to obtain one-to-one correspondence between surfaces without landmark constraints. A variety of algorithms have been developed to acquire these surface registrations, including shape information-based methods [6,7], conformal mapping-based methods which minimize the angle distortion $[8,9]$, and quasi-conformal mapping-based methods which minimize the maximal dilation of the mapping $[10,11]$.

Compared with landmark-free surface registration algorithms which cannot match landmark features, the landmark-constrained surface registration algorithms have been proposed to obtain a one-to-one correspondence between surfaces that matches landmark features. However, hard constraints may cause flipping in the mapping where diffeomorphism is not guaranteed. It is still a challenging problem to obtain a robust and bijective surface registration which can match landmarks consistently, especially when two surfaces undergo nonisometric large deformations.

Motivated by the above problem, we propose a novel method of robust registration for surfaces with large deformations and landmark constraints based on 
both optimal mass transport mapping (OMT-Map) and Teichmüller mapping (T-Map). The main idea behind this is to employ OMT-Map to achieve an area preserving mapping which maps source and target surfaces to a unit planar disk without large area distortion. Then the surface registration problem is converted to computing a landmark-matching T-Map between two planar disks such that the maximal conformality distortion is minimized while the mapping has uniform conformality distortion over the whole domain. In order to compute the T-Map efficiently and effectively, we propose an iterative algorithm in which an optimal Beltrami coefficient is obtained. The registration method proposed in this work is not only robust, but also guarantees diffeomorphism and landmark constraints for surfaces with large deformations.

\section{$1.1 \quad$ Related work}

In recent decades, 3D surface registration methods have been intensively investigated. Conformal mapping based surface registration methods $[12,13]$ map surfaces conformally to $2 \mathrm{D}$ canonical domains and boil 3D surface registration problem down to $2 \mathrm{D}$ image registration problem. These methods can minimize the angle distortion, while they may induce large area distortion. Quasi-conformal mapping based surface registration methods have also been proposed to tackle large-scale nonrigid deformations. The Beltrami holomorphic flow method [14] was presented to compute a unique surface quasi-conformal map. By adjusting Beltrami coefficients, surface diffeomorphisms are equivalently adjusted to obtain the optimal map with desired properties. In [15], a novel surface registration method based on quasi-conformal mapping was also proposed. This method extracts the features on the surfaces, then estimates the Beltrami coefficient, and finally uniquely determines the registration mapping by solving Beltrami equations using curvature flow. Compared with our proposed method, however, both conformal mapping based and quasi-conformal mapping based methods cannot avoid area distortion.

Landmark features are usually needed to guide surface registration. Landmarkconstrained surface registration has also been extensively studied. Among computer vision research efforts, an iterative algorithm based on Möbius Voting [16] was proposed to compute point correspondences between surfaces that are approximately partially isometric. However, it cannot guarantee the diffeomorphism. An optimization method based on landmark-constrained basis [17] was presented to establish full surface registration and geodesic deformation between two surfaces. In medical imaging field, [18] introduced a brain registration method using a quasi-exhaustive set of sulci as landmarks. [19] proposed an algorith$\mathrm{m}$ by extending landmark matching to the large deformation setting insuring the generation of diffeomorphisms, which was applied to brain registration. Recently, [20] proposed an OMT-Map based registration method. It composes the optimal mass transport map with the conformal map to obtain the unique area-preserving map, which is intrinsic to the Riemannian metric. This areapreserving map in conjunction with harmonic map was then used for surface registration, which avoids the large area distortion that conformal mapping may cause. Nevertheless, this method cannot guarantee the diffeomorphism. By con- 
trast, our proposed method can not only guarantee the diffeomorphism, but also is capable of minimizing the conformality distortion.

Compared with existing surface registration methods, e.g., the conformal mapping based method and OMT-Map based method, our proposed surface registration method is more advantageous: the OMT-Map which is employed in our method enforces the robustness by avoiding large area distortion; the TMap in our method produces the diffeomorphism with all the landmarks matched consistently.

\subsection{Contribution}

To the best of our knowledge, this work is the first one to propose the use of OMT-Map and T-Map to compute robust and unique bijective registration for surfaces with large deformations and landmark constraints. The major contribution of this work is the introduction of a novel surface registration framework based on the composition of T-Map with OMT-Map. It is advantageous due to the following merits:

- Robustness. The conventional conformal mapping-based surface registration method can preserve local shapes(i.e.,conformal) but may introduce large area distortions which exceed machine precision, resulting in problems and failure of surface registration. In contrast, our method is capable of constructing an area-preserving mapping which avoids large area distortion. Moreover, existing methods focus on registering near-isometric or near-conformal surfaces. In contrast, our method is capable of handling surfaces with large deformations, because of the flexibility of Teichmüller map. In theory, for any pair of homeomorphic surfaces with quite different geometries, there exists a unique Teichmüller map between them, which is diffeomorphic and minimizes the angle distortion. Thus our method is more robust.

- Diffeomorphism. In [20], the surface registration for landmark constraints is based on a harmonic mapping, which may encounter overlaps (flipping) although landmarks can be matched consistently. Thus, it is possible that diffeomorphism is not guaranteed. Compared with that method, this work proposes to compute a unique T-Map with landmark constraints such that diffeomorphism is guaranteed.

- Minimal conformality distortion. The algorithm for computation of T-Map in this work is to determine an optimal one-to-one correspondence for registration with landmark constraints. The computed T-Map can minimize the maximal conformality distortion and achieve uniform conformality distortion over the whole domain.

\section{Theoretic Background}

In this section, we briefly introduce the theoretic background. We refer readers to [21,22] for more details of optimal mass transport mapping and [23] for Teichmüller mapping. 


\subsection{Optimal Mass Transport Mapping}

The problem of optimal mass transport was first studied by Monge [24].

Definition 1 (Optimal Mass Transport Problem). Suppose $(X, \mu),(Y, \nu)$ represent two metric spaces $X$ and $Y$ with probabilities measures $\mu$ and $\nu$ respectively, satisfying the property of same total mass $\int_{X} \mu d x=\int_{Y} \nu d y . A$ map $T$ : $X \rightarrow Y$ is measure preserving if $\forall B \subset Y, \mu\left(T^{-1}(B)\right)=\nu(B)$. Let $c: X \times Y \rightarrow \mathbb{R}$ be a transportation cost function. The problem of optimal mass transport is to find the measure preserving map $T$ such that the following total transportation cost is minimized:

$$
\int_{X} c(x, T(x)) d \mu(x)
$$

Kantorovich [21] proposed the relaxation of Monge's problem and solved it by employing linear programming in the 1940's. Brenier [25] discovered and proved the connection between the optimal mass transport and the gradient map of a convex function at the end of 1980's.

\subsection{Discrete optimal mass transport mapping}

Our discrete optimal mass transport mapping is based on Brenier's approach.

Theorem 1 (Brenier). Suppose the transportation cost function c: $X \times Y \rightarrow \mathbb{R}$ is the quadratic Euclidean distance $c(x, y)=|x-y|^{2}$. If $\mu$ and $\nu$ have finite second order moments where $\mu$ is absolutely continuous, then there exists a convex function $u: X \rightarrow \mathbb{R}$ unique up to a adding a constant, such that the gradient map $\nabla u$ minimizes the quadratic transportation cost $\int_{X}|x-T(x)|^{2} d \mu(x)$, and gives the unique optimal mass transport mapping.

Let $\Omega$ be a convex domain in $X$ and space $Y$ be discretized to $\left\{y_{1}, y_{2}, \ldots, y_{k}\right\}$ with Dirac measure $\nu=\sum_{j=1}^{k} \nu_{j} \delta\left(y-y_{i}\right)$. Suppose a height vector consisting of $k$ real numbers is defined as $\mathbf{h}=\left(h_{1}, h_{2}, \ldots, h_{k}\right) \in \mathbb{R}^{n}$, and we define the supporting hyperplane for each $\left(y_{i}, h_{i}\right)$ as:

$$
\pi_{\mathbf{i}}(\mathbf{h}):\left\langle x, y_{i}\right\rangle+h_{i}=0
$$

where $\langle$,$\rangle denotes the inner product in \mathbb{R}^{n}$. A convex function $u_{\mathbf{h}}(x)$, representing the upper envelope of all supporting hyperplanes, is given by:

$$
u_{\mathbf{h}}(x)=\max _{1 \leq i \leq k}\left\{\left\langle x, y_{i}\right\rangle+h_{i}\right\}
$$

Let $G(\mathbf{h})$ denote the graph of $u_{\mathbf{h}}(x)$. The projection of $G(\mathbf{h})$ induces a polygonal partition of convex domain $\Omega$, i.e., $\Omega=\bigcup_{i=1}^{k} W_{i}(\mathbf{h})$, where each cell $W_{i}(\mathbf{h})$ is the projection of each facet of the convex polyhedron $G(\mathbf{h})$. The cell $W_{i}(\mathbf{h})$ is given by:

$$
W_{i}(\mathbf{h})=\left\{x \in X \mid u_{\mathbf{h}}(x)=\left\langle x, y_{i}\right\rangle+h_{i}\right\} \cap \Omega
$$

and its area is defined as:

$$
w_{i}(\mathbf{h})=\int_{W_{i}(\mathbf{h})} \mu(x) d x
$$




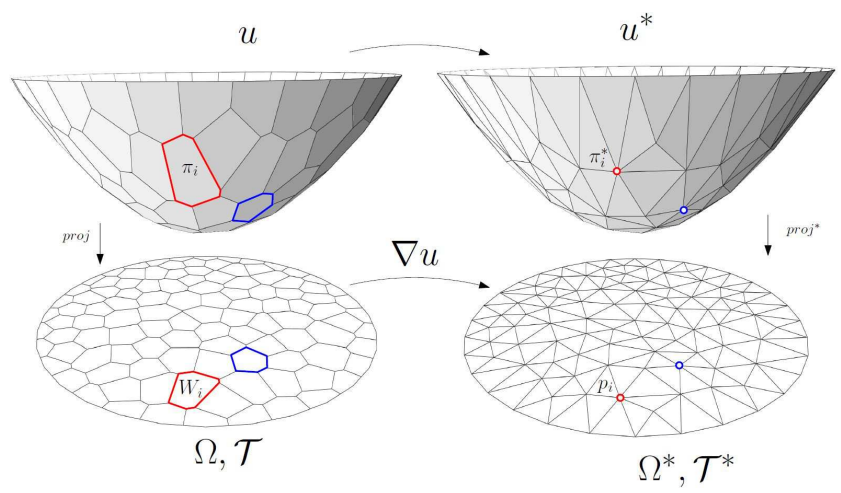

Fig. 1. Discrete optimal mass transport mapping.

Since the convex function $u_{\mathbf{h}}(x)$ on each cell $W_{i}(\mathbf{h})$ is a linear function $\pi_{\mathbf{i}}(\mathbf{h})$, the gradient map $\nabla u_{\mathbf{h}}$ given below maps each cell $W_{i}(\mathbf{h})$ to a single point $y_{i}$ :

$$
\left.\nabla u_{\mathbf{h}}\right|_{W_{i}(\mathbf{h})}=y_{i}
$$

where $i=1,2, \ldots, k$. The cell decomposition induced by a convex function $u$ is illustrated in Figure 1, with details explained in [22].

An important theorem serving as a theoretic foundation for discrete optimal mass transport theory and computational algorithm is also presented as follows:

Theorem 2. For any given measure $\nu$, which satisfies $\sum_{j=1}^{k} \nu_{j}=\int_{\Omega} \mu$ where $\nu_{j}>0$, there must exist a height vector $\mathbf{h}$ unique up to adding a constant vector, and the convex function $u_{\mathbf{h}}(x)=\max _{1 \leq i \leq k}\left\{\left\langle x, y_{i}\right\rangle+h_{i}\right\}$ induces the cell decomposition of $\Omega, \Omega=\bigcup_{i=1}^{k} W_{i}(\mathbf{h})$, such that each cell satisfies the area-preserving constraint as follows:

$$
\int_{W_{i}(\mathbf{h})} \mu(x) d x=\nu_{i}, i=1,2, \ldots, k .
$$

In addition, the gradient map $\nabla u_{\mathbf{h}}$ minimizes the quadratic transportation cost below:

$$
\int_{\Omega}|x-T(x)|^{2} \mu(x) d x
$$

Alexandrov [26] first proved the existence and uniqueness, and Brenier [25] also proved the uniqueness and optimality. Recently, Gu et al. [22] have proposed a novel proof for the existence and uniqueness using the variational principle. We follow their approach in our work.

The admissible space of height vectors is defined as follows:

$$
H_{0}:=\left\{\mathbf{h} \mid \Sigma_{j=1}^{k} h_{j}=0, \int_{W_{i}(\mathbf{h})} \mu>0, \forall i=1, \ldots, k\right\}
$$

According to Brunn-Minkowski theorem [26], $H_{0}$ is a convex domain. We can construct a convex energy function

$$
E(\mathbf{h})=\int_{0}^{\mathbf{h}} \sum_{i=1}^{k} w_{i}(\eta) d \eta_{i}-\sum_{i=1}^{k} \nu_{i} h_{i}+C
$$


where $C$ is a constant. Note that the first term in above equation is the volume of the convex polyhedron bounded by the graph $G(\mathbf{h})$ and the cylinder comprising vertical lines through boundary of $\Omega$.

Thus, the gradient of the energy function is denoted by

$$
\nabla E(\mathbf{h})=\left(w_{1}(\mathbf{h})-\nu_{1}, \ldots, w_{k}(\mathbf{h})-\nu_{k}\right)^{T}
$$

Let $e_{i j}=W_{i}(\mathbf{h}) \cap W_{j}(\mathbf{h}) \cap \Omega$ be the edge where the cells $W_{i}(\mathbf{h})$ and $W_{j}(\mathbf{h})$ intersect. Then Hessian of the energy function $E(\mathbf{h})$ is expressed as:

$$
\frac{\partial^{2} E(\mathbf{h})}{\partial h_{i} \partial h_{j}}= \begin{cases}\frac{\int_{e_{i j}} \mu(x) d x}{\left|y_{j}-y_{i}\right|} & W_{i}(\mathbf{h}) \cap W_{j}(\mathbf{h}) \cap \Omega \neq \emptyset \\ 0 & \text { otherwise. }\end{cases}
$$

$\mathrm{Gu}$ et al. [22] have proven that the admissible space $H_{0}$ is convex and the energy in Eqn.(10) is hence convex, when $\Omega$ is convex. Moreover, the unique global minimizer $h_{0}$ is an interior point of $H_{0}$, which induces the unique optimal mass transport mapping.

The global minimizer can be computed efficiently using Newton's method due to the convexity of the energy in Eqn.(10). In comparison to Kantorovich's approach where $n^{2}$ variables are unknown, this approach has only $n$ unknown variables.

\subsection{Teichmüller mapping}

As a special class of diffeomorphisms, Teichmüller mapping (T-Map) ia a quasiconformal mapping with uniform conformality distortion over the whole domain. We give some theoretic foundation relevant to Teichmüller mapping below.

Definition 2 (Conformal Mapping). Let $(S, g)$ be a surface $S$ with a Riemannian metric $g$. A diffeomorphism $f:(S, g) \rightarrow\left(\mathbb{D}, d x^{2}+d y^{2}\right)$ maps the surface to a planar unit disk $\mathbb{D} . f$ is a conformal(angle-preserving) mapping, if $g(x, y)=e^{2 \lambda(x, y)}\left(d x^{2}+d y^{2}\right)$, where $\lambda: S \rightarrow \mathbb{R}$ is called conformal factor which denotes the area distortion.

Quasi-conformal mapping is a generalization of conformal mapping, which preserves orientation between Riemann surfaces with bounded conformality distortions. We give its definition as follows:

Definition 3 (Quasi-conformal Mapping). Suppose $f: \mathbb{C} \rightarrow \mathbb{C}$ is a diffeomorphism. $f$ is a quasi-conformal mapping if $f$ satisfies the Beltrami equation:

$$
\frac{\partial f}{\partial \bar{z}}=\mu(z) \frac{\partial f}{\partial z}
$$

for some complex-valued function $\mu: \mathbb{C} \rightarrow \mathbb{C}$ satisfying $\|\mu\|_{\infty}<1 . \mu$ is called the Beltrami coefficient, which measures the local conformality distortion of $f$.

The quasi-conformal mapping maps infinitesimal circles to infinitesimal ellipses. As shown in Figure 2, the ratio between the longer axis and the shorter axis of the infinitesimal ellipse is denoted by $\frac{1+|\mu|}{1-|\mu|}$, which describes the eccentricity of the infinitesimal ellipse. The orientation of infinitesimal ellipse is given by the angle between the longer axis and the real axis, i.e., $\theta=\frac{1}{2} \arg \mu$. The maximal dilation of $f$ is given by:

$$
K(f)=\frac{1+\|\mu\|_{\infty}}{1-\|\mu\|_{\infty}}
$$




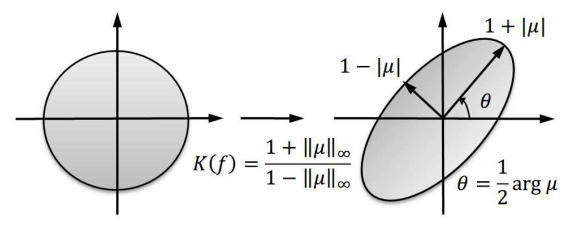

Fig. 2. Geometric illustration of Beltrami coefficient $\mu$.

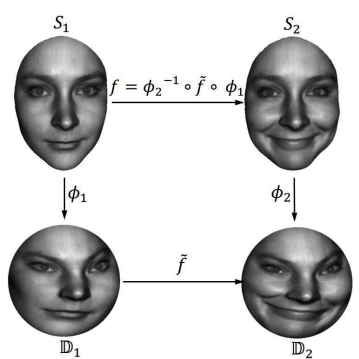

Fig. 3. Overall framework of our registration algorithm.

In terms of the quasi-conformal mapping between two Riemann surfaces, we use Beltrami differential rather than Beltrami coefficient. A Beltrami differential $\mu(z) \frac{d \bar{z}}{d z}$ on a Riemann surface is an assignment to each chart $\left(U_{\alpha}, \phi_{\alpha}\right)$ of an $L_{\infty}$ complex-valued function $\mu_{\alpha}$, which is defined on parameter $z_{\alpha}$ such that:

$$
\mu_{\alpha}\left(z_{\alpha}\right) \frac{d \bar{z}_{\alpha}}{d z_{\alpha}}=\mu_{\beta}\left(z_{\beta}\right) \frac{d \bar{z}_{\beta}}{d z_{\beta}}
$$

on the domain covered by another chart $\left(U_{\beta}, \phi_{\beta}\right)$. Here, $\frac{d z_{\beta}}{d z_{\alpha}}=\frac{d \phi_{\alpha \beta}}{d z_{\alpha}}$ and $\phi_{\alpha \beta}=$ $\phi_{\beta} \circ \phi_{\alpha}^{-1}$.

A T-Map is intuitively a quasi-conformal map with uniform distribution of conformality distortions. We give the definition of T-Map as follows:

Definition 4 (Teichmüller Mapping). Suppose $f: S_{1} \rightarrow S_{2}$ is a quasiconformal mapping. $f$ is a Teichmüller mapping associated with the integrable holomorphic function $\varphi: S_{1} \rightarrow \mathbb{C}$ if its associated Beltrami coefficient is of the form:

$$
\mu(f)=k \frac{\bar{\varphi}}{|\varphi|}
$$

for some constant $0 \leq k<1$ and holomorphic function $\varphi \neq 0$ with $\|\varphi\|_{1}=$ $\int_{S_{1}}|\varphi|<\infty$. The Beltrami coefficient of this form is of Teichmüller type.

T-Map can be utilized for surface registration with landmark constraints. We can obtain a unique T-Map with landmark constraints by computing an optimal Beltrami coefficient such that the maximal dilation is minimized and the norm of Beltrami coefficient is constant everywhere.

\section{Algorithms}

In this section, the OMT-Map based area preserving mapping algorithm is presented with details, followed by the T-Map algorithm. Finally, the proposed surface registration framework is given.

\subsection{OMT-Map based area preserving mapping algorithm}

Suppose $(S, g)$ is a simply connected surface $S$ with Riemannian metric $g$. According to Riemann mapping theorem, there exists a conformal mapping $\phi:(S, g) \rightarrow(\mathbb{D}, d z d \bar{z})$ such that $g=e^{2 \lambda(z)} d z d \bar{z}$. The conformal factor $\lambda$ determines a measure $\nu=e^{2 \lambda(z)} d z d \bar{z}$ on the unit disk $\mathbb{D}$. Then there exists a 
Algorithm 1 OMT-Map based Area Preserving Mapping

Input: A triangle mesh $M$ with disk topology and a threshold $\delta w$ of area difference.

Output: An area preserving mapping $f: M \rightarrow \mathbb{D}$ where $\mathbb{D}$ is a planar unit disk.

1: Compute a conformal mapping $\phi: M \rightarrow \mathbb{D}$ based on the holomorphic one-form method.

2: Initialize height vector $\mathbf{h} \leftarrow(0,0, \ldots, 0)$, and set target area $\bar{w}_{i} \leftarrow \nu_{i}$ using Eqn.(17). Then translate and scale all points in $P$ such that they are all in the unit disk $\mathbb{D}$.

3: Compute the power diagram, then compute the area $w_{i}$ of each cell $W_{i}$ and the edge lengths.

4: Compute the dual power Delaunay triangulation and edge lengths.

5: Construct the Hessian matrix $H$ according to Eqn.(12).

6: Update the height vector $\mathbf{h} \leftarrow \mathbf{h}+H^{-1}(\overline{\mathbf{w}}-\mathbf{w})$.

7: Repeat step 3 through step 6 until $\left\|\bar{w}_{i}-w_{i}\right\|<\delta w$ for each cell. Then we obtain a OMT-Map $\tau:(\mathbb{D}, d z d \bar{z}) \rightarrow(P, \nu)$.

8: The area preserving mapping is constructed by $\tau^{-1} \circ \phi: M \rightarrow \mathbb{D}$, where each vertex $v_{i} \in M$ is mapped to the centroid $c_{i}$ of cell $W_{i}$.

unique OMT-Map $\tau:(\mathbb{D}, d z d \bar{z}) \rightarrow(\mathbb{D}, \nu)$. The area preserving mapping is then given by the composition $\tau^{-1} \circ \phi:(S, g) \rightarrow(\mathbb{D}, d z d \bar{z})$.

In the discrete algorithm, the surface $S$ is approximated by a triangle mesh $M$ with scaled total area $\pi$. The conformal mapping $\phi:(M, g) \rightarrow(\mathbb{D}, d z d \bar{z})$ is computed based on the holomorphic one-form method [27]. All vertices $v_{1}, v_{2}, \ldots, v_{n} \in$ $M$ are mapped conformally onto unit disk $\mathbb{D}$, and form a point set which is denoted by $P=\left\{\phi\left(v_{1}\right), \phi\left(v_{2}\right), \ldots, \phi\left(v_{n}\right)\right\}$. In the discrete OMT-Map $\tau:(\mathbb{D}, d z d \bar{z}) \rightarrow$ $(P, \nu)$, the discrete measure for $\nu$ is defined as follows:

$$
v_{i}=\frac{1}{3} \sum_{\left[v_{i}, v_{j}, v_{k}\right] \in M} \operatorname{Area}\left(\left[v_{i}, v_{j}, v_{k}\right]\right)
$$

where $\left[v_{i}, v_{j}, v_{k}\right]$ is a triangle face adjacent to $v_{i}$.

The discrete OMT-Map based area preserving mapping can be computed by minimizing the convex energy in Eqn.(10) using Newton's method. The algorithm is illustrated in Alg.1.

\subsection{Teichmüller mapping algorithm}

The T-Map is computed using an iterative algorithm closely related to computation of harmonic mapping. The algorithm iteratively solves Beltrami equation to obtain an optimal Beltrami coefficient which corresponds to the desired T-Map.

Specifically, a landmark-constrained harmonic mapping $f^{(k)}: \mathbb{D}_{1} \rightarrow \mathbb{D}_{2}$ is computed based on current discrete metric [28], where $k=1,2, \ldots$ The Beltrami coefficient $\mu$ of above mapping is then computed according to Beltrami equation in Eqn.(13). To obtain a Beltrami coefficient of the Teichmüller type, we project above Beltrami coefficient $\mu$ into the one with constant norm as follows:

$$
\mathcal{P}(\mu)=\left(\frac{\int_{\mathbb{D}_{1}}|\mu| d \mathbb{D}_{1}}{\int_{\mathbb{D}_{1}} d \mathbb{D}_{1}}\right) \frac{\mu}{|\mu|}
$$




\section{Algorithm 2 Teichmüller Mapping}

Input: Two triangulated planar unit disks $\mathbb{D}_{1}, \mathbb{D}_{2}$, two corresponding interior landmark constraints $\left\{p_{i}\right\}_{i=1}^{n} \in \mathbb{D}_{1},\left\{q_{i}\right\}_{i=1}^{n} \in \mathbb{D}_{2}$, and a threshold $\delta \mu$ of Beltrami coefficient difference.

Output: A T-Map $\widetilde{f}: \mathbb{D}_{1} \rightarrow \mathbb{D}_{2}$ which matches landmarks

1: Compute initial edge length and edge weight in $\mathbb{D}_{1}$, and set $k \leftarrow 0$.

2: Set $k \leftarrow k+1$, and then compute a constrained harmonic map $f^{(k)}: \mathbb{D}_{1} \rightarrow \mathbb{D}_{2}$, such that $f^{(k)}$ aligns the corresponding landmarks given above.

3: Compute the Beltrami coefficient $\mu$ defined on each face using Eqn.(13).

4: Compute a projection $\mathcal{P}(\mu)$ on Beltrami coefficient $\mu$ using Eqn.(18).

5: Smooth the projection $\mathcal{P}(\mu)$ and obtain the updated Beltrami coefficient $\mu^{\prime}$.

6: Deform the metric in original source disk $\mathbb{D}_{1}$ using above updated Beltrami coefficient $\mu^{\prime}$ by computing each new edge length and edge weight.

7: Repeat step 2 through step 6 until $\left\|\mu^{\prime}-\mu\right\|<\delta \mu$ for each face. The final T-Map is given by $\tilde{f}=f^{(k)}$.

\section{Algorithm 3 Landmark-constrained Surface Registration}

Input: Triangular meshes of surfaces $S_{1}$ and $S_{2}$ with disk topology.

Output: A one-to-one correspondence $f: S_{1} \rightarrow S_{2}$ which registers the source surface to the target surface.

1: Locate a set of corresponding points on $S_{1}$ and $S_{2}$ respectively as the landmark constraints.

2: Use Alg.1 to compute the OMT-Map based area preserving mapping $\phi_{i}: S_{i} \rightarrow \mathbb{D}_{i}$, where $i=1,2$ and $\mathbb{D}_{i}$ is a unit disk.

3: Use Alg.2 to compute a landmark-constrained T-Map $\tilde{f}: \mathbb{D}_{1} \rightarrow \mathbb{D}_{2}$.

4: The surface registration with landmark constraints is given by $f=\phi_{2}^{-1} \circ \tilde{f} \circ \phi_{1}$ : $S_{1} \rightarrow S_{2}$.

The projection $\mathcal{P}(\mu)$ is then smoothed, becoming the updated Beltrami coefficient $\mu^{\prime}$ which is used to deform the discrete metric in original disk $\mathbb{D}_{1}$.

The above procedure continues until the iteration converges. As a result, the Beltrami coefficient $\mu$ converges to an optimal one of Teichmüller type, which induces the corresponding T-Map $\tilde{f}: \mathbb{D}_{1} \rightarrow \mathbb{D}_{2}$. Furthermore, the maximal dilatation is minimized and the norm of Beltrami coefficient is constant everywhere. The algorithm for computation of this T-Map is given by Alg.2.

\subsection{Surface registration algorithm}

We combine the T-Map with OMT-Map for surface registration with landmark constraints. The geometric illustration of the overall framework is shown in Figure 3. The Alg.3 gives the details of the landmark-constrained surfaces registration algorithm.

\subsection{Computational complexity}

Our proposed algorithm includes three steps, i.e., conformal mapping, optimal mass transport mapping and Teichmüller mapping. For the first step, there are 


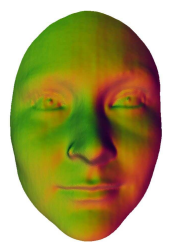

(a) Source surface

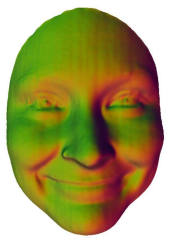

(e) Target surface

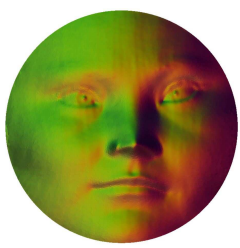

(b) Conformal map

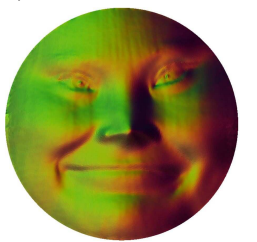

(f) Conformal map

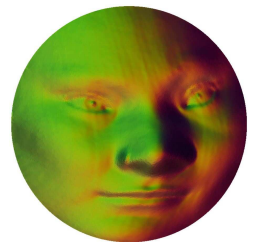

(c) OMT-Map

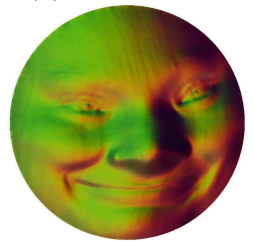

(g) OMT-Map

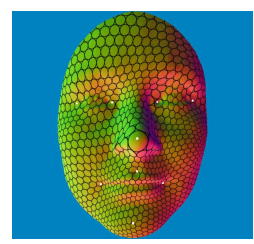

(d) Texture mapping

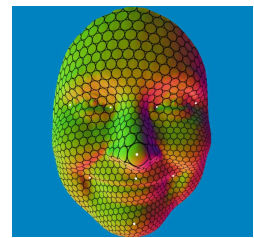

(h) Texture mapping

Fig. 4. Surface mapping results of Sophie model. (a-c).source surface, its conformal mapping result, and OMT-Map result; (e-g).target surface, its conformal mapping result, and OMT-Map result; (d\&h). T-Map from source surface in (d) to target surface in $(\mathrm{h})$, which is illustrated by texture mapping.

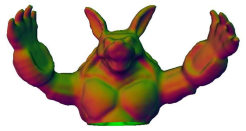

(a) Source surface

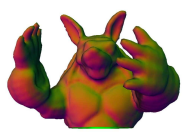

(e) Target surface

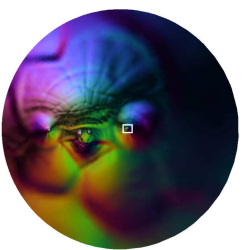

(b) Conformal map

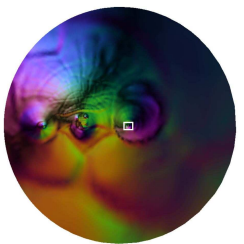

(f) Conformal map

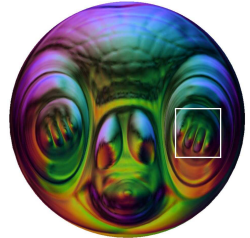

(c) OMT-Map

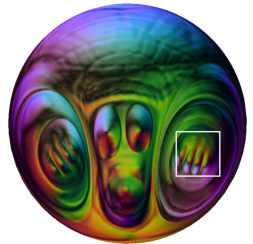

(g) OMT-Map

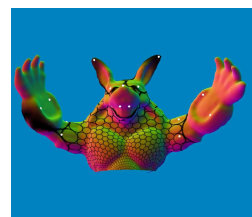

(d) Texture mapping

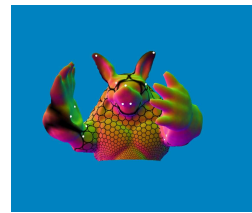

(h) Texture mapping

Fig. 5. Surface mapping results of Armadillo model. (a-c).source surface, its conformal mapping result, and OMT-Map result; (e-g).target surface, its conformal mapping result, and OMT-Map result; (d\&h). T-Map from source surface in (d) to target surface in $(\mathrm{h})$, which is illustrated by texture mapping.

different algorithms for computing conformal mappings, and their complexities are comparable. For the second step, there are mainly two approaches, i.e., Kontarovich's method and Brenier's approach. Our method is based on Brenier's approach, which involves $O(n)$ unknown variables. In contrast, Kantorovich's approach has $O\left(n^{2}\right)$ unknowns. For the third step, our method can compute the Teichmüller maps between surfaces with landmark constraints efficiently. 


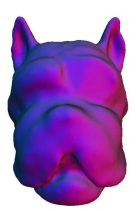

(a) Source surface

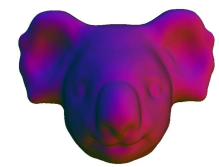

(e) Target surface

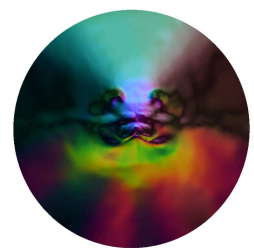

(b) Conformal map

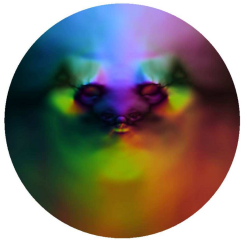

(f) Conformal map

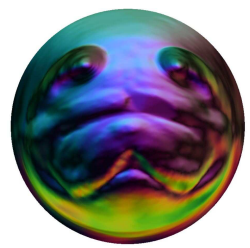

(c) OMT-Map

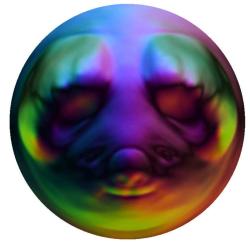

(g) OMT-Map

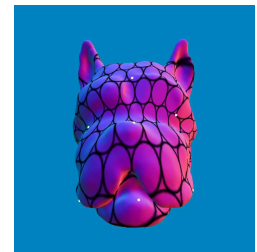

(d) Texture mapping

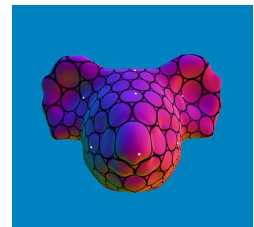

(h) Texture mapping

Fig. 6. Surface mapping results of Dog and Koala bear models. (a-c).source surface, its conformal mapping result, and OMT-Map result; (e-g).target surface, its conformal mapping result, and OMT-Map result; (d\&h). T-Map from source surface in (d) to target surface in $(\mathrm{h})$, which is illustrated by texture mapping.

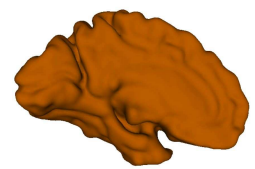

(a) Source surface

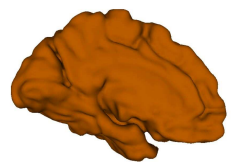

(e) Target surface

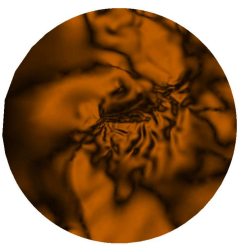

(b) Conformal map

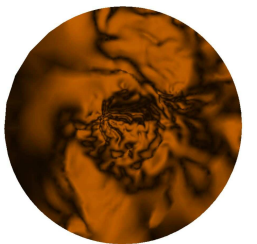

(f) Conformal map

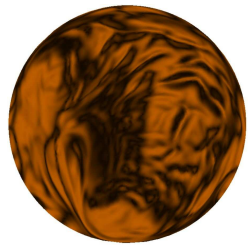

(c) OMT-Map

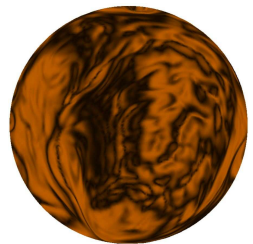

(g) OMT-Map

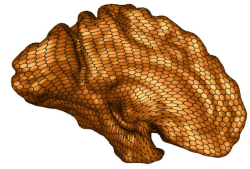

(d) Texture mapping

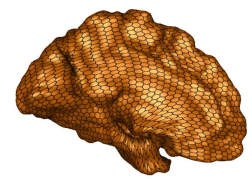

(h) Texture mapping

Fig. 7. Surface mapping results of brain. (a-c).source surface, its conformal mapping result, and OMT-Map result; (e-g).target surface, its conformal mapping result, and OMT-Map result; (d\&h). T-Map from source surface in (d) to target surface in (h), which is illustrated by texture mapping.

\section{Experiments}

We implemented our algorithms using $\mathrm{C}++$ on Windows platform, and evaluated our landmark-constrained registration method by comparing it with existing registration methods, i.e., conformal mapping based method [13] and OMTMap based method [20]. The experimental evaluation was performed on various surface models and a collection of 10 brain surfaces shown in Table 1, where the geometric properties of surface model (e.g., number of vertices, edges, and faces) 


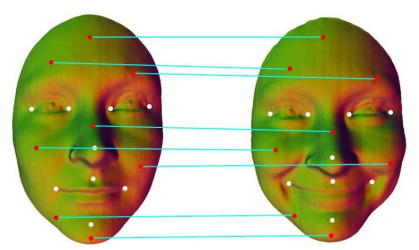

(a) Sophie model

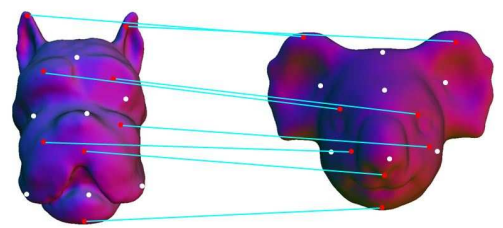

(c) Dog and Koala models

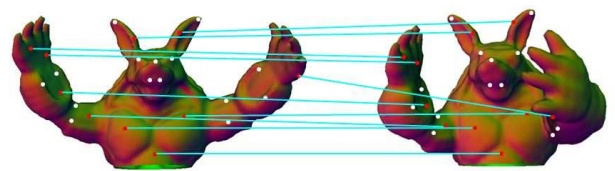

(b) Armadillo model

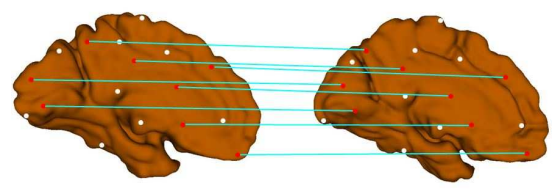

(d) Brain surfaces

Fig. 8. Surface registration results. The registered correspondences by the proposed method are shown for (a). Sophie model; (b). Armadillo model; (c). dog and koala bear models; (d). brain surfaces. The white circular dots denote the landmark constraints, and the cyan lines connecting the red circular dots between the source and target surfaces demonstrate the registered correspondences.

and landmarks information were reported. All the experiments were conducted on a laptop computer of Intel Core i7 CPU, 2.60GHz with $8 \mathrm{~GB}$ memory.

Figures 4, 5, 6 and 7 show the surface mapping results for Sophie model, Armadillo model, Dog and Koala bear models, as well as some brain surfaces, respectively. In the above figures, the subfigure (a) gives the original source surface, and the subfigures (b) and (c) represent the conformal mapping result and OMT-Map result of the source surface. Similarly, the subfigure (e) shows the target surface in the registration, and the subfigures (f) and (g) demonstrate the conformal mapping result and OMT-Map result of the target surface. Illustrated by the texture mappings in the subfigures (d) and (h), the T-Map gives the registration result of our proposed method which maps circle-texture in subfigure (d) to ellipse-texture in subfigure (h), with landmark constraints denoted by white dots. Moreover, Figure 8 illustrates the registration results of our proposed method. The white circular dots denote the landmark constraints, and the cyan lines connecting the red circular dots demonstrate the registered correspondences between the source and target surfaces. In Figure 9, the texture mapping results are given to qualitatively show the registration accuracy of our proposed method.

\subsection{Robustness}

The proposed landmark-constrained registration method employs the OMTMap, which is capable of avoiding large area distortion. To evaluate the robustness of our method, we demonstrate the conformal mapping results and 


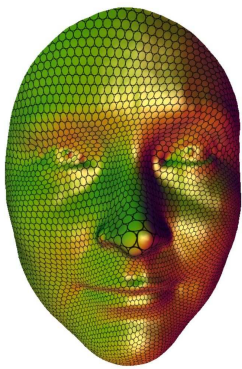

(a)

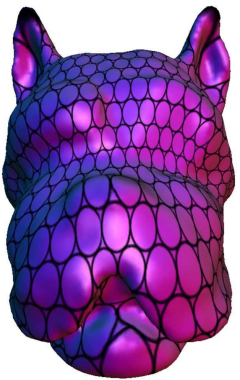

(c)

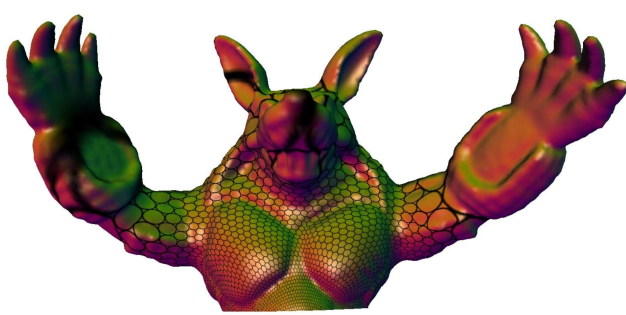

(b)

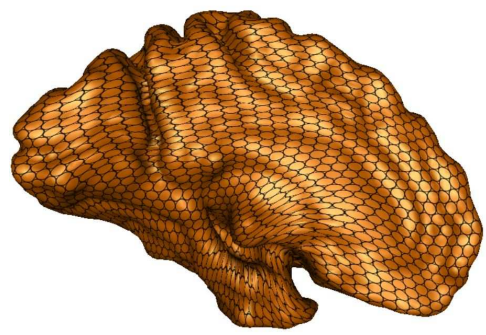

(d)

Fig. 9. Texture mapping results: (a). Sophie model; (b). Armadillo model; (c). dog model; (d). brain surface.

OMT-Map results for Armadillo surface models. The Armadillo surfaces were mapped onto the planar unit disk by conformal mapping and OMT-Map, respectively. As observed from Figure 5(b) and (f) which illustrate the conformal mapping results, the head area shrunk exponentially and became hard to be recognized. In the region marked with small white box, the hand with fingers suffered from exponential area distortion, which may easily exceed the machine precision and result in problems or failure of surface registration. By contrast, the fingers marked with a bigger white box in Figure 5(c) and (g) where the OMT-Map based area preserving mapping results are shown, maintained the same areas as those on the original Armadillo surface. The similar observation was also obtained from two different mapping results for dog and koala bear surface models, where the ear and nose of conformal mapping results in Figure 6(b) and (f) are respectively shrunk with large area distortion, which are nevertheless avoided in OMT-Map based area preserving mapping results in Figure 6(c) and $(\mathrm{g})$. Therefore, the OMT-based area preserving mapping in our registration method avoids large area distortion, rendering our registration method more robust.

\subsection{Diffeomorphism}

One merit which makes our registration method advantageous is that the diffeomorphism of the mapping between surfaces is guaranteed. To quantitatively 

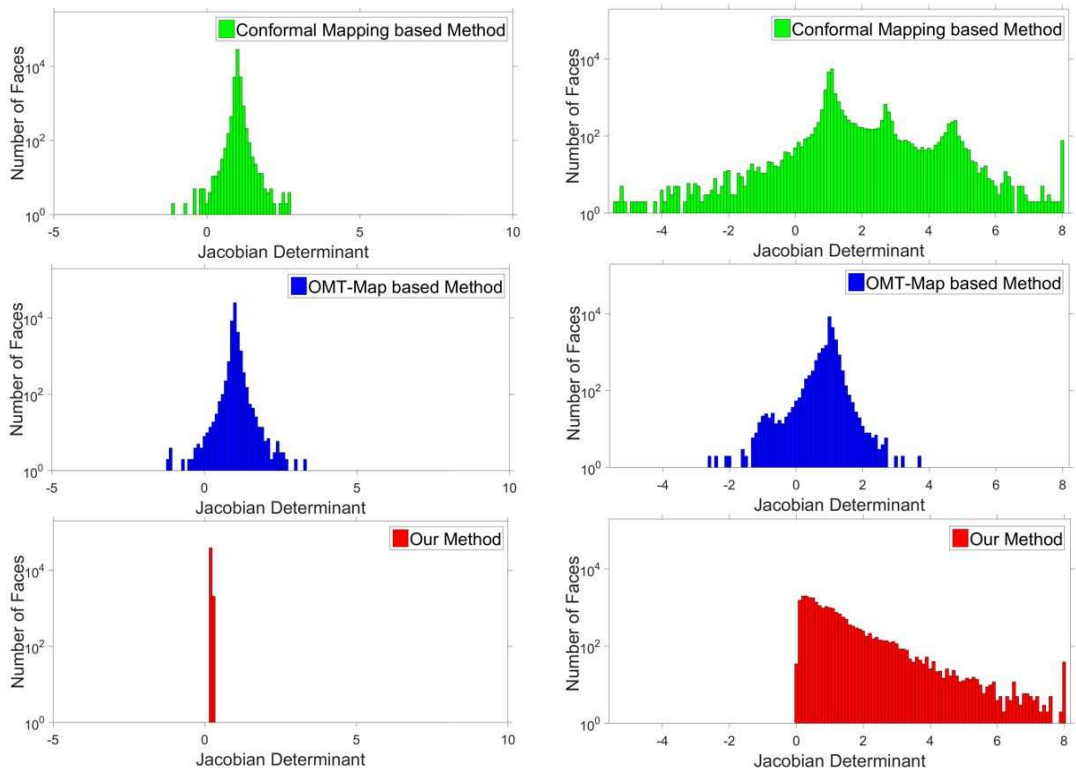

(a) Result of Sophie model

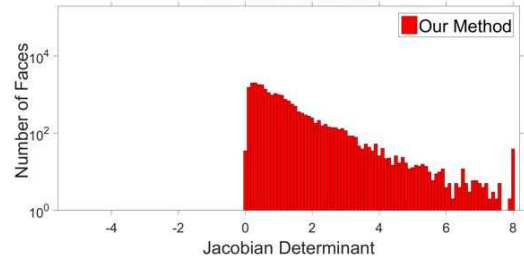

(b) Result of Armadillo model
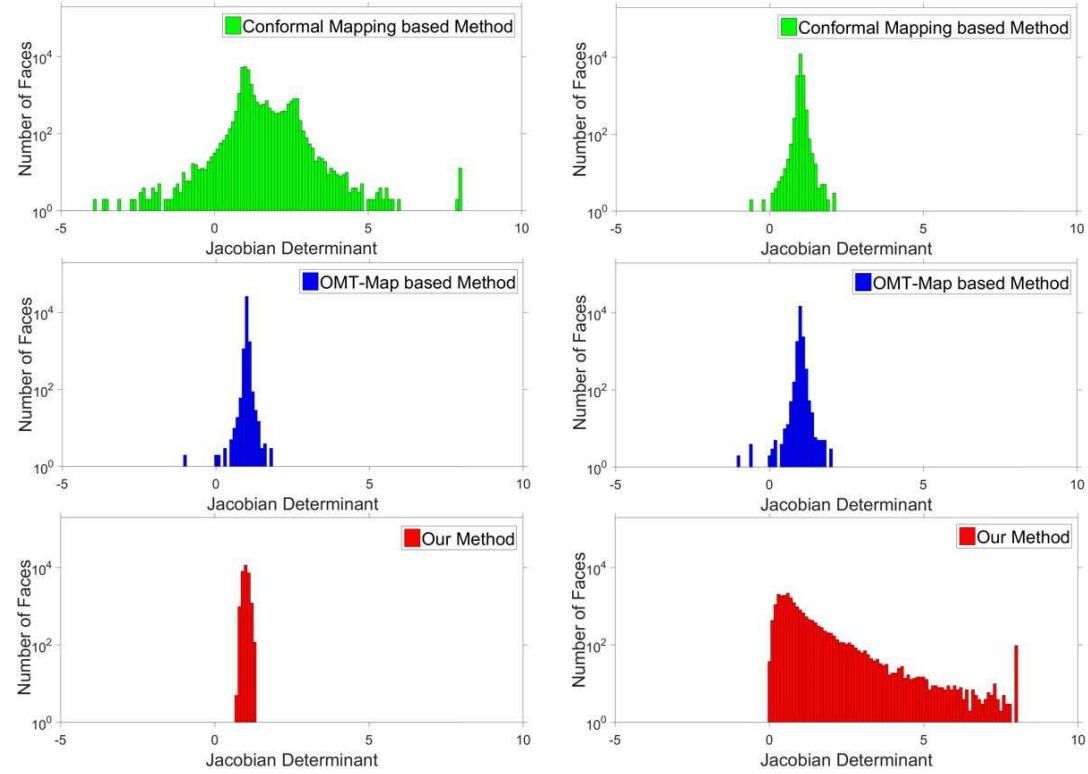

(c) Result of Dog and Koala models

(d) Result of brain surfaces

Fig. 10. Comparison results of three methods on histograms of Jacobian determinant for various surfaces in Table 1. The number of negative Jacobian determinants corresponds to the number of flipped faces. The diffeomorphism is indicated by zero flipped face. 
Table 1. Geometric properties of surface models.

\begin{tabular}{lcccc}
\hline Surface Model & \#Vertices & \#Edges & \#Faces & \#Landmarks \\
\hline Sophie\#1 & 21043 & 62629 & 41587 & 9 \\
Sophie\#2 & 21495 & 64012 & 42518 & 9 \\
\hline Armadillo\#1 & 11251 & 33654 & 22404 & 15 \\
Armadillo\#2 & 10933 & 32702 & 21770 & 15 \\
\hline Dog & 14858 & 44423 & 29566 & 10 \\
Koala bear & 13768 & 41137 & 27370 & 10 \\
Source brain surface & $10 \mathrm{k} \sim 20 \mathrm{k}$ & $30 \mathrm{k} \sim 60 \mathrm{k}$ & $20 \mathrm{k} \sim 40 \mathrm{k}$ & 10 \\
Target brain surface & $10 \mathrm{k} \sim 20 \mathrm{k}$ & $30 \mathrm{k} \sim 60 \mathrm{k}$ & $20 \mathrm{k} \sim 40 \mathrm{k}$ & 10 \\
\hline
\end{tabular}

Table 2. Comparison of three methods on diffeomorphism.

\begin{tabular}{lccc}
\hline Number of flipped faces & Conformal Map & OMT-Map & Ours \\
\hline \#Flipped faces for Sophie model & 27 & 38 & 0 \\
\#Flipped faces for Armadillo model & 564 & 302 & 0 \\
\#Flipped faces for dog\&koala bear model & 227 & 7 & 0 \\
\#Flipped faces for brain surfaces in average & 7 & 14 & 0 \\
\hline
\end{tabular}

evaluate the registration performance in guaranteeing the diffeomorphism, we compared our registration method with the other two existing methods, i.e., conformal mapping based method and OMT-Map based method. Specifically, we computed the Jacobian determinant, and measured the number of flipped faces by counting the number of negative Jacobian determinants, for various surface models as well as a set of brain surfaces. Zero flipped faces indicate that the diffeomorphism in the surface registration result is obtained. As shown in Table 2, our proposed method guarantees the diffeomorphism by obtaining zero flipped faces in the registration result, while both of the other two existing methods produced some flipped faces, where diffeomorphisms were not guaranteed. In Figure 10.(a)(b)(c)(d) which capture the experimental results for the Sophie, Armadillo, Dog and Koala bear, and the brain surfaces respectively, the upper frame with green bars and the middle frame with blue bars indicate that overlaps (i.e., flipped regions) occur in the registration results of the other two existing methods, respectively. On the contrary, there is no flipped faces in the registration result of our proposed registration method, as evidenced by the lower frame with red bars in Figure 10.(a)(b)(c)(d). Thus, it is shown that our registration method is effective in computing a non-overlap landmark-constrained registration, and guarantee the diffeomorphism.

\subsection{Conformality distortion}

Our registration method can compute a unique T-Map with constrained landmarks. In order to evaluate the proposed registration method, we computed the norm of Beltrami coefficient which measures the local conformality distortion, and compared our registration method with the other two existing methods for various surfaces. As illustrated in the upper frames and the middle frames of Figure 11.(a)(b)(c)(d), we obtained the maximal conformality distortions $|\mu|_{\max }>1$ for both of the two existing methods. By contrast, our method achieved $|\mu|_{\text {max }}$ $=0.27<1,|\mu|_{\max }=0.66<1,|\mu|_{\max }=0.32<1$, and $|\mu|_{\max }=0.59<1$ for the Sophie, Armadillo, Dog and Koala bear, and the brain surfaces respectively. 

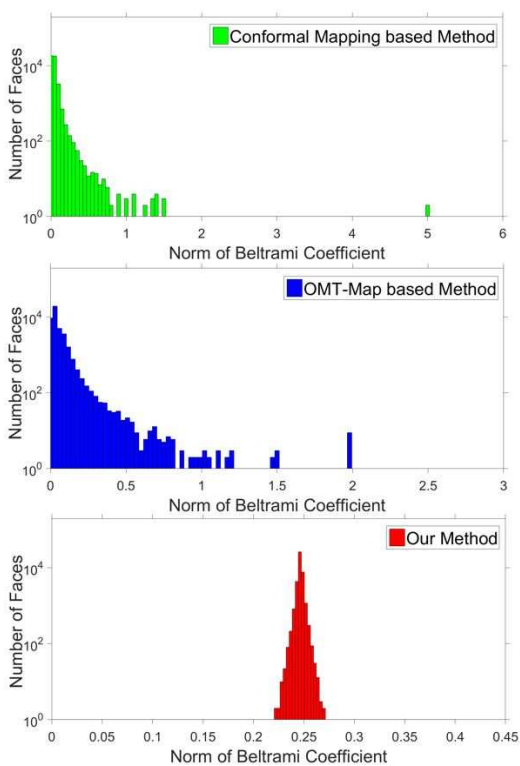

(a) Result of Sophie model
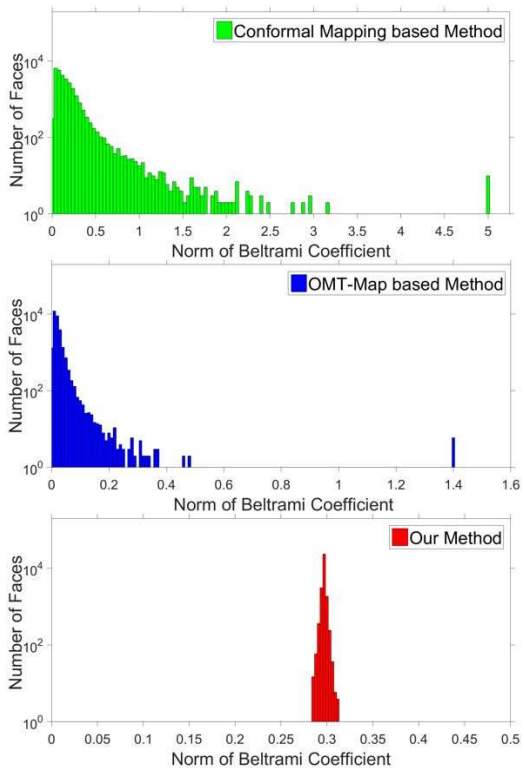

(c) Result of Dog and Koala models
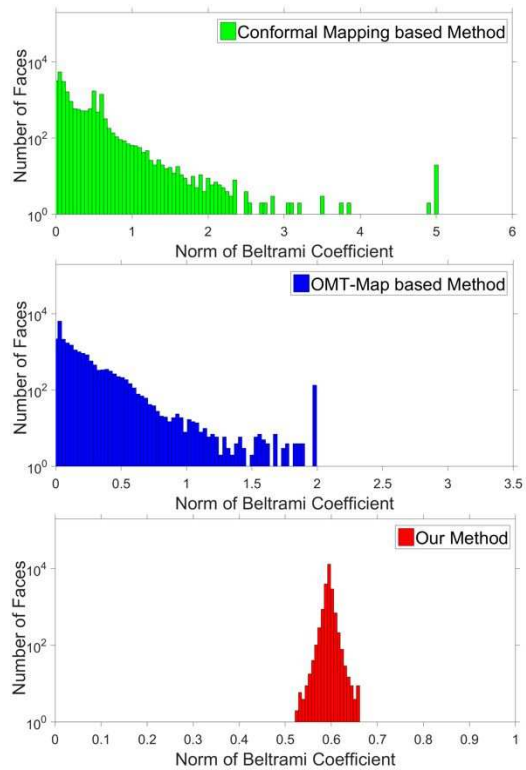

(b) Result of Armadillo model
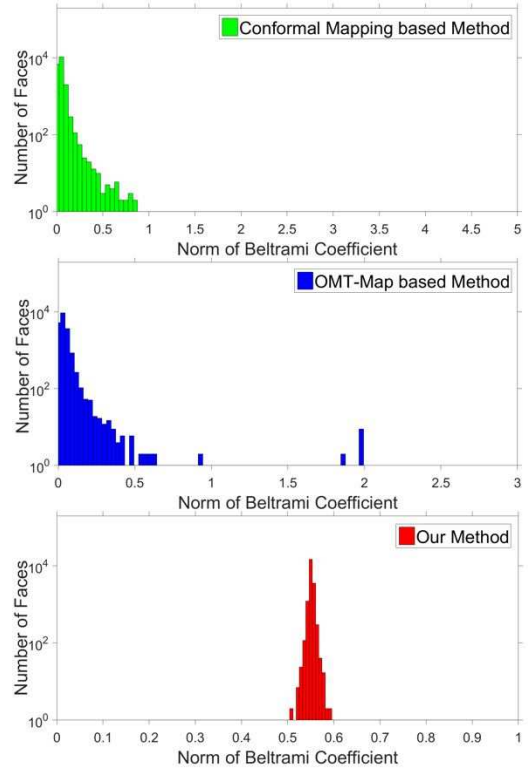

(d) Result of brain surfaces

Fig. 11. Comparison results of three methods on conformality distortion for various surfaces in Table 1. The upper frame and middle frame in each sub-figure show the results of the conformal mapping based method and the OMT-Map based method, respectively. The lower frame gives the results of our proposed method. 

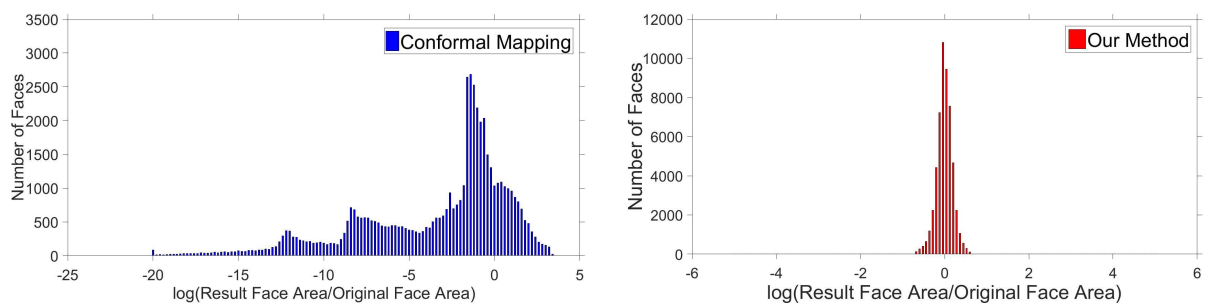

Fig. 12. Comparison results of our method with conformal mapping based method on area distortion.

niformly equal to $0.24,0.59,0.29$, and 0.55 respectively, as given in the lower frames of Figure 11.(a)(b)(c)(d). This demonstrates our registration method can minimize the maximal conformality distortion and achieve uniform conformality distortion over the whole domain.

\subsection{Area Distortion}

In order to demonstrate the advantage of our proposed method on avoiding large area distortion, we conducted numerical evaluation on Armadillo models by comparing our method with the existing conformal mapping based method. Figure 12 shows the the histograms of area distortion, which is computed by the logarithm of result face area over the original face area. As observed from the Figure 12(a), the existing conformal mapping based method causes large shrinkage and area distortion. In contrast, our method almost keeps the same area and avoids large area distortion in Figure 12(b).

\subsection{Comparision with Teichmüller Map based Method}

We further compared our method with the pure Teichmüller map (T-Map) based method [29]. Both methods were run with the same convergence threshold in the implementation on Armadillo models. According to the experimental results, both methods achieved diffeomorphism and obtained zero flipped face in the registration results. However, our method, in terms of the running time, performed better with a value of 51 seconds, comparing with the T-Map based method with a value of 88 seconds. Moreover, the norms of Beltrami coefficient were computed. As shown in Figure 13(a), the distribution of the norm of Beltrami coefficient in the T-Map based method is not even. By contrast, nearly all the norms of Beltrami coefficient in our method is uniformly distributed at around 0.59 in Figure 13(b).

\section{Conclusion}

In this work, a robust and diffeomorphic landmark-constrained surface registration method is proposed based on OMT-Map and T-Map. The algorithm$\mathrm{s}$ for computation of OMT-Map based area preserving mapping, T-Map and 

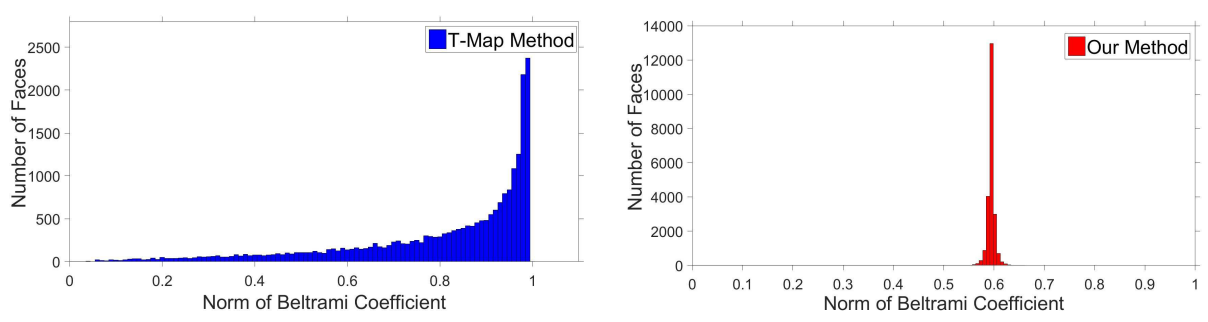

Fig. 13. Comparison results of our method with the Teichmüller map based method on conformality distortion.

landmark-based surface registration framework are presented with details. Experimental evaluations are performed on real surface data, which shows that our registration method is robust, accurate and diffeomorphic. Current work focuses on simply connected surfaces. In the future, we will generalize the framework to surfaces with more general topology.

\section{Acknowledgements}

This work was supported by the funding sources NSF DMS-1418255, NSF DMS1221339, and AFOSR FA9550-10-1-0294.

\section{References}

1. Wyngaerd, J., Gool, L., Koch, R., Proesmans, M.: Invariant-based registration of surface patches. In ICCV (1999) 301-306

2. Huber, D., Kapuria, A., Donamukkala, R., Hebert, M.: Parts-based 3d object classification. In CVPR (2004)

3. Wang, S., Wang, Y., Jin, M., Gu, X., Samaras, D.: Matching and recognition using conformal geometry. In CVPR (2) (2006) 2453-2460

4. Yeo, B.T., Sabuncu, M.R., Vercauteren, T., Ayache, N., Fischl, B., Golland, P.: Spherical demons:fast diffeomorphic landmark-free surface registration. IEEE Transactions on Medical Imaging 29(3) (2010) 650-668

5. Lui, L., Thiruvenkadam, S., Wang, Y., Thompson, P., Chan, T.: Optimized conformal surface registration with shape-based landmark matching. SIAM Journal of Imaging Sciences 3(1) (2010) 52-78

6. Lord, N.A., Ho, J., Vemuri, B.C., Eisenschenk, S.: Simultaneous registration and parcellation of bilateral hippocampal surface pairs for local asymmetry quantification. IEEE Transactions on Medical Imaging 26(4) (2007) 471-478

7. Lyttelton, O., Boucher, M., Robbins, S., Evans, A.: An unbiased iterative group registration template for cortical surface analysis. NeuroImage 34 (2007) 15351544

8. Gu, X., Wang, Y., Chan, T.F., Thompson, P.M., Yau, S.T.: Genus zero surface conformal mapping and its application to brain surface mapping. IEEE Transactions on Medical Imaging 23(8) (2004) 949-958 
9. Hurdal, M.K., Stephenson, K.: Discrete conformal methods for cortical brain flattening. NeuroImage 45 (2009) 86-98

10. Weber, O., Myles, A., Zorin, D.: Computing extremal quasiconformal maps. Computer Graphics Forum 31(5) (2012) 1679-1689

11. Zeng, W., Lui, L.M., Luo, F., Chan, T.F., Yau, S.T., Gu, X.: Computing quasiconformal maps using an auxiliary metric and discrete curvature flow. Numerische Mathematik 121(4) (2012) 671-703

12. Bronstein, A.M., Bronstein, M.M., Kimmel, R.: Generalized multidimensional scaling: A framework for isometry-invariant partial surface matching. In Proceedings of National Academy of Science (2006) 1168-1172

13. Zeng, W., Samaras, D., Gu, X.: Ricci flow for 3d shape analysis. IEEE TPAMI 32(4) (2010) 662-677

14. Lui, L.M., Wong, T.W., Zeng, W., Gu, X., Thompson, P.M., Chan, T.F., Yau, S.T.: Optimization of surface registrations using beltrami holomorphic flow. Journal of Scientific Computing 50(3) (2012) 557-585

15. Zeng, W., Gu, X.: Registration for 3d surfaces with large deformations using quasi-conformal curvature flow. In CVPR (2011)

16. Lipman, Y., Funkhouser, T.: Möbius voting for surface correspondence. ACM Transactions on Graphics 28(3) (2009)

17. Kurtek, S., Srivastava, A., Klassen, E., Laga, H.: Landmark-guided elastic shape analysis of spherically-parameterized surfaces. Computer Graphics Forum 32 (2013) 331-349

18. Auzias, G., Colliot, O., Glaunes, J.A., Perrot, M., Mangin, J.F., Trouve, A., Baillet, S.: Diffeomorphic brain registration under exhaustive sulcal constraints. IEEE Transactions on Medical Imaging 30(6) (2011) 1214-1227

19. Joshi, S.C., Miller, M.I.: Landmark matching via large deformation diffeomorphisms. IEEE Trans Image Process 9(8) (2000) 1357-1370

20. Su, Z., Wang, Y., Shi, R., Zeng, W., Sun, J., Luo, F., Gu, X.: Optimal mass transport for shape matching and comparison. IEEE TPAMI (2015)

21. Kantorovich, L.V.: On a problem of monge. Uspekhi Mat. Nauk. 3 (1948) 225-226

22. Gu, X., Luo, F., Sun, J., Yau, S.T.: Variational principles for minkowski type problems, discrete optimal transport, and discrete monge-amperé equations. arXiv:1302.5472 (2013) 1-13

23. Gardiner, F., Lakic, N.: Quasiconformal teichmüller theory. American Mathematics Society (2000)

24. Bonnotte, N.: From knothes rearrangement to breniers optimal transport map. arXiv:1205.1099 [math.OC] (2012) 1-29

25. Brenier, Y.: Polar factorization and monotone rearrangement of vectorvalued functions. Comm. Pure. Appl. Math. 44(4) (1991) 375-417

26. Alexandrov, A.D.: Convex polyhedra. Springer Monographs in Mathematics (2005)

27. Gu, X., Yau, S.T.: Computing conformal structures of surfaces. Communications In Information and Systems 2(2) (2002) 121-146

28. Wang, Y., Gu, X., Hayashi, K.M., Chan, T.F., Thompson, P.M., Yau, S.T.: Surface parameterization using riemann surface structure. In ICCV 2 (2005) 1061-1066

29. Zeng, W., Ming Lui, L., Gu, X.: Surface registration by optimization in constrained diffeomorphism space. In: Proceedings of the IEEE Conference on Computer Vision and Pattern Recognition. (2014) 4169-4176 


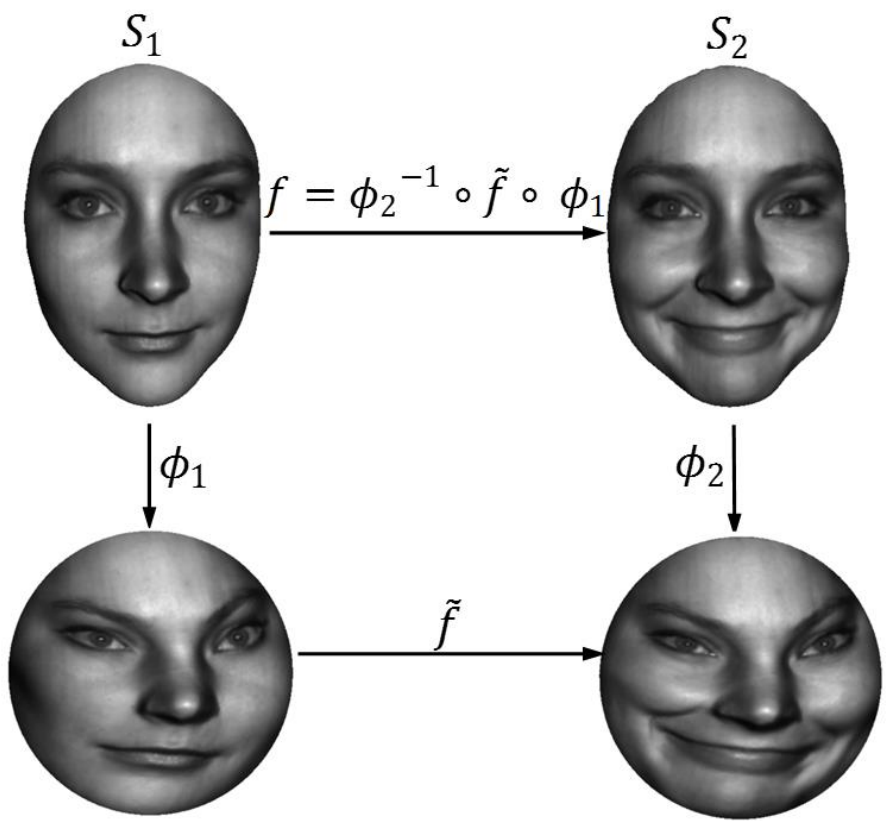

$\phi_{1}$ and $\phi_{2}$ are optimal mass transport based area preserving mappings. $\tilde{f}$ is the Teichmüller mapping. $f$ is the resulting mapping for surface registration. 